\title{
LA SUISSE
}

L'une des particularités de la proscription communaliste en Suisse par rapport à celle des autres pays, c'est d'être, pour une bien plus large part, issue des mouvements qui soulevèrent diverses villes de province. Cela signifie qu'elle n'a pas débuté au lendemain de la Semaine sanglante, mais bien avant déjà, au fur et à mesure qu'échouaient les différentes Communes provinciales. Le décalage chronologique entre l'insurrection parisienne et les mouvements qui agitèrent le reste du pays fit qu'au moment où celle-ci résistait encore victorieusement, toute une série de réfugiés de Lyon, du Creusot, de Marseille ou d'ailleurs se morfondaient à Genève, prêts à saisir la première occasion pour rentrer dans leur pays et y déclencher un nouveau soulèvement.

Cette situation allait être exploitée par la Commune de Paris pour essayer de desserrer l'étreinte versaillaise en suscitant de nouveaux mouvements en province. Mais, pour cela, la présence des réfugiés à Genève ne suffisait pas; fallait-il encore qu'ils y trouvassent l'aide nécessaire pour mener à bien leurs projets. Celle-ci ne pouvait leur être fournie que par les deux tendances de l'Internationale existant en Suisse et par une petite frange de radicaux plus ou moins liés aux sections genevoises de l'A.I.T. C'est pourquoi il nous faut examiner tout d'abord les relations qui existaient, avant les événements, entre les futurs communeux de France et leurs compagnons en Suisse.

\section{La Suisse et ses réfugiés en 1871}

Dès ses débuts, l'Internationale en Suisse avait eu des relations très étroites avec les sections françaises. Les trois congrès de l'A.I.T. qui s'étaient tenus en Suisse, le soutien mutuel des grèves dans les deux pays, les liens traditionnels unissant Genève et Lyon, le rôle des étrangers et des réfugiés politiques en Suisse, tout cela avait facilité les contacts de part et d'autre de la frontière. L'action de Bakounine, depuis 1868, avait également noué, entre ses partisans, un véritable réseau de relations qui joua pleinement au lendemain de la scission de la 
Fédération romande, au printemps 1870 . Juste auparavant, prévoyant la victoire de ses adversaires entre les mains desquels resterait l'Egalité, Bakounine écrivait à Albert Richard, à Lyon, pour dénoncer le rédacteur du journal romand, son compatriote Nicolas Outine:

"Il en profitera naturellement pour se mettre en rapport avec le socialisme français. Mon cher, je te demande donc énergiquement, impérieusement, au nom de toute notre Intimité, de prévenir tous nos amis de France et surtout Mme D. T. [Varlin], Mme D. Z. [Bastelica], Mr. E. [Aubry] et Mr. D. U. [Malon] sans oublier Mr. D. Z. [Palix] de cette sale et réactionnaire intrigue. Outine doit être mis au ban de notre intimité, comme un être malfaisant; et tous les bons, tous ceux qui marchent avec nous, soit directement soit indirectement, doivent s'en garder comme d'une peste. Car son intrigue est insinuante, perfide, dissolvante. Avertis tous ceux qui se trouvent sous ton influence ou sous celle de nos amis. Si notre parti succombe, la séparation et l'organisation indépendante des sections des Montagnes aura lieu et alors nous aurons pour organe non l'Egalité, mais le Progrès du Locle, qui passera probablement alors à Neufchâtel, sous la direction de Guillaume. Ce sera alors le Progrès que nous devrons soutenir par nos correspondances et en lui procurant beaucoup d'abonnements.» ${ }^{1}$

Ce désir d'amener les sections françaises de l'Internationale à peser de tout leur poids en faveur des «jurassiens» et "alliancistes» et à priver les adversaires de ceux-ci de leurs relations internationales est attesté par d'autres documents. ${ }^{2}$ La manœuvre réussit d'autant plus facilement que les adversaires de l'Alliance semblent avoir négligé d'informer les autres pays; ainsi César de Paepe à Bruxelles aussi bien qu'Aubry à Rouen se plaignirent de ne plus recevoir l'Egalité. ${ }^{3}$ Les sections françaises avaient donc plus de contacts avec les militants de l'Alliance qu'avec les dirigeants genevois de la Fédération romande.

Cependant, la guerre, la coupure des communications et la désagrégation de l'organisation de l'Internationale, en Suisse comme en France, entraînèrent un relâchement de ces relations. Quand James

1 Genève, $1^{\text {er }}$ avril 1870, Archives municipales de la Ville de Lyon, I² 56. En fait, le Progrès sera remplacé par la Solidarité qui parut à Genève et non à Neuchâtel, comme organe de la Fédération romande de la tendance Bakounine.

2 Cf. la lettre de Bakounine à Joukowski, 6 avril 1870, in: Max Nettlau, Michael Bakunin. Eine Biographie (The Life of Michael Bakounine), Londres 1896-1900, pp. 393-394. Aubry, cependant, adopta une position originale, pleine de réserve à l'égard des Genevois, mais très critique envers leurs adversaires (La Réforme sociale, 8 mai 1870 ).

${ }^{3}$ L'Internationale, 5 juin 1870; La Réforme sociale, 29 mai 1870. 
Guillaume publia, au lendemain du 4 Septembre, son manifeste appelant ses compatriotes à voler au secours de la République française, il le fit sans consultation préalable des Français ni même de ses compagnons jurassiens. ${ }^{1}$ L'équipée de Bakounine à Lyon et ses suites obligèrent ses partisans à se disperser et, dans une certaine mesure, les déconsidérèrent; néanmoins, les relations avec la Suisse se renouèrent; suivant une vieille habitude, remontant à 1831 pour le moins, plusieurs des participants aux troubles de l'automne 1870 à Lyon se réfugièrent à Genève. Gaspard Blanc, Albert Richard, Saignes y séjournèrent plus ou moins longtemps, semble-t-il. ${ }^{2}$ Au début de mars 1871, au dire d'Albert Leblanc, des relations existaient entre Lyon et Genève, maintenues par l'intermédiaire d'Albert Richard. ${ }^{3}$ Cluseret, lui aussi, s'était réfugié à Genève après le 28 septembre. Il y vécut assez retiré, n'ayant de contacts, à ses dires, qu'avec Jules Barni, un républicain qui avait refusé le serment imposé par Napoléon III aux universitaires et qui avait été nommé professeur de philosophie à l'Académie de Genève; avec l'avocat Amberny, radical en étroites relations avec l'Internationale, et avec deux dirigeants de celle-ci: Outine et le Français Duval, ouvrier menuisier et ancien maréchal des logis aux chasseurs d'Afrique. A la fin du mois d'octobre, un délégué de Marseille, le cordonnier Maviel, était venu lui proposer le commandement des forces militaires de la future Commune phocéenne. Cluseret se mit aussitôt en route, accompagné jusqu'à Annecy par Outine et Duval qui, quelques heures après avoir pris congé de leur protégé, furent arrêtés et interrogés par le préfet Jules Philippe, lequel avait été alerté par le consul de France à Genève. Après l'échec du mouvement marseillais, en novembre, Cluseret se cacha et, à une date indéterminée, revint à Genève où sa présence est attestée au début de mars 1871 ; c'est bien de Suisse et non

1 D'où, sans doute, certaines réactions négatives auxquelles semble répondre la lettre de Bakounine "Aux amis du Conseil fédéral des Sections de l'Internationale de la Suisse romande à la Chaux-de-Fonds", datée de Genève, le 13 septembre 1870 (Le Réveil, 21 août 1926).

${ }^{2}$ Le consul de France à Genève prétend qu'un chef de l'association de la CroixRousse de Lyon, nommé Cottin, serait venu s'entendre avec Cluseret pour déclencher un nouveau mouvement. Mais ce dernier et Saignes auraient fait de nombreuses réserves, exigeant la constitution préalable de dépôts d'armes, l'adoption du drapeau rouge, etc. (Archives du Ministère des Affaires Etrangères, Paris, Correspondance politique des consuls, Genève, vol. 9, 24 février 1871). Information à accueillir avec prudence, le consul ayant été à plusieurs reprises la dupe de ses informateurs.

3 Albert Leblanc à Constant Martin, Lyon, 3 mars 1871, in: Jean Dautry et Lucien Scheler, Le Comité central républicain des vingt arrondissements de Paris, Paris 1960, p. 206.

4 Mémoires du Général Cluseret, Paris 1887-1888, t. II, pp. 100 et 102-103. 
de Saint-Etienne, comme il le prétendra, qu'il partira pour se mettre au service de la Commune de Paris. ${ }^{1}$

L'échec du mouvement lyonnais du 23 au 25 mars fit refluer vers Genève plusieurs dizaines de ses participants, qui y entrèrent à nouveau en relations avec les deux tendances de l'Internationale. Peu après, se formait, au sein des sections genevoises, un comité secret, dans le but de venir en aide au mouvement communaliste. Il se composait uniquement d'adversaires de Bakounine. ${ }^{2}$ Ses contacts avec la France se faisaient par l'intermédiaire de quelques correspondants et, surtout, par celui des réfugiés. C'est sans doute ce groupe qui, sous le nom de "Comité révolutionnaire des provinces", publia un Appel aux républicains dévoués resté ignoré des historiens jusqu'à aujourd'hui. ${ }^{3}$

C'est sur ces entrefaites qu'arriva à Genève Jean-Louis Artus, un enfant du pays fixé depuis quelques années à Paris. Ardent radical, sympathisant avec les républicains avancés, il s'était engagé dans la Garde nationale où il avait été élu délégué lors de sa constitution en fédération. Envoyé en mission d'information et de propagande par Paschal Grousset, le délégué aux relations extérieures, Artus prit contact avec le comité de Genève et repartit pour Paris vers le 27 avril. ${ }^{4}$

Pendant ce temps, les préparatifs se poursuivaient pour relancer le mouvement à Lyon; Albert Leblanc faisait la navette entre les deux villes. ${ }^{\mathbf{5}}$ Le 22 avril, Colonna, réfugié à Genève après les journées de mars, lui envoyait la lettre suivante:

1 Sa présence à Genève, en février-mars 1871, est attestée par la lettre de Leblanc citée plus haut. Mais, dans ses Mémoires, Cluseret prétend être parti de SaintEtienne pour rejoindre Paris au lendemain du 18 Mars. Sans doute voulait-il dissimuler le fait, connu des autorités françaises, qu'il avait, pour gagner la capitale depuis la Suisse, traversé le territoire occupé par les Allemands.

2 Voir en annexe le document II.

3 On en trouvera le texte ci-dessus, publié par M. Moissonnier (document VI en annexe à son article). Tout confirme ses hypothèses. Ainsi les caractères typographiques sont absolument identiques à ceux qu'employait habituellement l'Imprimerie coopérative d'où sortaient les publications des sections genevoises et de la Fédération romande. Les caractères employés habituellement par l'imprimerie Czerniecki, où les partisans de Bakounine faisaient tirer la Solidarité, sont tout différents.

4 M. Vuilleumier, "L'Internationale à Genève et la Commune de Paris, 1871", in: Mélanges offerts à M. Paul E. Martin, Genève 1961, pp. 625-643. Paru également in: Mémoires et documents publiés par la Société d'histoire et d'archéologie de Genève, t. XL.

s C'est ce que laisse supposer le faux passeport suisse qu'il utilisait et qui porte les mentions suivantes: "Vu à Genève le 21 avril 1871. Bon pour Lyon + retour à Genève avant le 30 courant. Vu à Genève 29 avril 1871. Bon pour Lyon.» Archives Départementales du Rhône (A.D.R.), R, 1 er Conseil de guerre, dossiers individuels 1871-1872, Insurrection de la Guillotière, 30 avril 1871, dossier 
"Citoyen Leblanc,

Je vous prie de m'accuser réception par le même courrier, les dispositions d'esprit sur la garde nationale de Lyon [sic], et savoir quels sont les éléments qui manquent pour la Commune, nous avons dans l'Internationale des travailleurs de Genève, tous les éléments voulus, nous attendons les réponses des délégués de Lyon, de Savoie et de Var, pour pouvoir agir, tous à la fois, nous devons recevoir 500 chassepots et cartouches, nous devons proclamer la Commune de partout où nous passerons, jusqu'aux villes désignées ci-dessus, en partant 500, nous arriverons par milliers de cette manière, les forces du gouvernement illusoire de Versailles, sera obligé [sic] de diviser sa force.

Je ne puis vous en dire d'avantage, je suis obligé de mettre la lettre à la poste.

Salut et fraternité Colonna»

A en croire la minute de sa réponse, Leblanc approuva chaudement et demanda l'itinéraire pour pouvoir avertir ses amis du passage de la colonne. Il est vrai qu'à l'instruction de son procès, il déclarera avoir renoncé à envoyer sa réponse à cause du caractère provocateur de la lettre de Colonna. ${ }^{1}$ Le moins qu'on puisse dire est que ce dernier avait commis une grave imprudence.

Le 24 avril, Caulet de Tayac, envoyé par la Commune, écrivait à Dupont: "Je suis à Lyon depuis trois jours; j'y suis arrivé après avoir traversé la Suisse où j'espère avoir déterminé un mouvement qui se traduira par un manifeste de l'Internationale et de toutes les sociétés ouvrières en général en faveur de la Commune de Paris. A Lyon, malgré

Albert Leblanc. Il est vrai que le passeport aurait pu également être utilisé par une autre personne!

1 A.D.R., ibid. La lettre de Colonna fut transmise par l'intermédiaire de Jean Coignet, dit Joanny (ibid. pièces générales, réquisitoire). Elle fut lue au procès et le Journal de Genève la publia par deux fois, les 4 et 12 novembre 1871, avec le brouillon de la réponse de Leblanc. Colonna écrivit à la presse; il reconnaissait l'authenticité de sa lettre, "mais j'affirme énergiquement que mes lettres sont restées sans réponse, et que ni Albert Leblanc et Coignet ne m'ont jamais écrit. J'affirme en outre n'avoir jamais vu ni parlé à Albert Leblanc lors de son prétendu voyage qu'il aurait fait à Genève" (La Suisse radicale, 14 novembre 1871; Journal de Genève, 15 novembre 1871). Ce dernier quotidien ajoutait: "Il reste donc établi qu'au printemps dernier, tout était préparé dans le sein de l'Internationale à Genève pour une incursion armée sur le territoire d'un pays voisin et ami, incursion destinée à renverser le gouvernement régulier et à venir en aide à la Commune révolutionnaire de Paris. Or $\mathrm{M}$. Colonna pouvait-il ignorer les conséquences extrêmement graves que son entreprise devait avoir pour le pays qui lui donnait alors l'hospitalité?" 
un certain découragement, issu fatalement de l'insuccès de mars, l'élément révolutionnaire ne fait pas défaut. J'y ai retrouvé Leblanc et Dumont, antérieurement délégués par la Commune, qui ont beaucoup et bien travaillé. Nous espérons faire le mouvement dans le courant de la semaine, et des délégués des centres ouvriers de la région de Tarare, St Rambert etc., nous promettent de se lever à notre premier signal.» ${ }^{1}$

Cependant, les efforts pour soutenir Lyon se firent en ordre dispersé. Quand Leblanc revint à Genève, sans doute le 27 ou le 28 avril, il semble avoir surtout pris contact avec les «alliancistes». Selon Outine, ce serait Ozeroff qui aurait joué le double jeu et aurait amené le délégué de la Commune à collaborer avec les amis de Bakounine. ${ }^{2}$ La chose fut d'autant plus facile que ce dernier comptait d'assez nombreux adeptes chez les Lyonnais. Pour préparer la journée du 30 avril, on fit imprimer des affiches chez Czerniecki, affiches dont Joukowski corrigea les épreuves d'une manière déplorable, y laissant de nombreuses coquilles. ${ }^{3}$ Une des affiches, intitulée Commune de Lyon, était un appel à soutenir Paris et annonçait la formation d'une Commune provisoire dans la cité rhodanienne. D'après Perret et Outine, les signatures y avaient été apposées sans consultation des intéressés. ${ }^{4}$

Mais la police française était informée des intentions de Leblanc, comme le prouve cette lettre du commissaire spécial de Bellegarde au préfet du Rhône, le 29 avril 1871: "Je m'empresse de vous faire connaître que, conformément à votre dépêche télégraphique expédiée de Lyon ce matin à 10 heures 40 minutes, j'ai pris les dispositions nécessaires pour assurer l'arrestation du n[omm]é Albert Leblanc signalé comme devant rentrer en France porteur de trois affiches de la Commune imprimées à Genève. Ayant passé en revue tous les voyageurs arrivés à Bellegarde par le train 252, parti de Genève à 5 h. 20 minutes du soir, j'ai cru reconnaître dans la personne de l'un d'eux l'individu

1 Voici la suite de la lettre: "Leblanc et Dumont ont fondé un journal le Cri du Peuple lyonnais, qui se lit et accélérera le mouvement. Maintenant nous sommes un peu paralysés par le manque de fonds; passez donc aux Finances et envoyez m'en par la personne qui viendra vous trouver au nom de Leblanc. Répétez à la Commune ce que je vous écris et dites à Viard que Dumont, qui l'attendait à Lyon, espère qu'il lui fera également concéder par la Commune un surcroît de subsides. Au revoir, cher Dupont, faites immédiatement parvenir votre réponse (après démarche aux Finances) à Melle Leblanc, 42, rue Turbigo. Salut fraternel. Signé: Caulet de Tayac. Salut aux amis.» Archives de la Préfecture de Police, Paris, B/a 1003, dossier Caulet de Tayac. On trouve tantôt de Tayac, tantôt du Tayac.

2 Cf. document VI.

3 J. Guillaume, L'Internationale. Documents et souvenirs (1864-1878), Paris 1905-1910, t. II, p. 174.

4 A.D.R., ibid., dossier Leblanc. Les signataires du texte sont Rivoire, Bergeron, Brugnal, G. Blanc, Bouret, Tacussel, Pelea, Velay, Audouard. 
signalé en question, bien qu'il eût substitué un chapeau jaune à son chapeau noir, qu'il ne fût plus porteur du cache-nez mentionné dans le télégramme et que ses moustaches ne soient point coupées en brosse." Après s'être assuré qu'aucun comparse ne l'accompagnait, le commissaire le prit à part et le fit fouiller. ${ }^{1}$ Il était porteur d'un passeport genevois établi au nom de John Benoit, un typographe de Genève qui dirigeait l'Imprimerie coopérative d'où sortaient les publications de l'Internationale. Par mesure de précaution, Leblanc avait sur lui diverses lettres adressées à Benoit, pour le cas où l'on aurait mis en doute sa fausse identité. ${ }^{2}$ Il était armé d'un revolver chargé et d'une poire de poivre. On trouva, dans ses vêtements, 90 affiches de la Commune de Lyon, 200 de l'appel de la Commune de Paris aux départements et 11 numéros de la Solidarité du 25 avril, qui publiait en supplément la Déclaration de la Commune de Paris au peuple français datée du 19 avril. «Nous n'avons pu trouver la troisième proclamation mentionnée par la dépêche télégraphique», ajoutait le commissaire de Bellegarde, décidément bien renseigné. Sans doute était-elle emportée par Camille Camet, un jeune canut lyonnais, qui, lui, sut échapper à l'arrestation et arriva à destination avec ses affiches. ${ }^{3}$ Selon Andrieux, d'autres arrestations auraient été opérées le même jour: "plusieurs émissaires dont le départ m'avait été signalé» et, à Lyon, Payet et Codex. ${ }^{4}$

Désorganisé par ces mesures policières préventives, le mouvement partit fort mal et ne tarda pas à échouer. James Guillaume, ayant appris le soulèvement du 30 avril, gagna aussitôt Genève pour s'y concerter avec Perron et Joukowski. Le 2 mai, alors qu'il s'apprêtait à prendre le train, il apprit l'échec du mouvement et renonça à partir. ${ }^{5}$ Tous ces épisodes confirment le jugement sévère de Duval, délégué par le comité de Genève à Lyon, et justifient, dans une très large mesure, les accusations d'Henri Perret. ${ }^{6}$

1 A.D.R., M, dossier Correspondance de De Gourlet, commissaire central puis directeur de la Sûreté générale à Lyon avec les commissaires spéciaux de Ferney et Bellegarde. Non classé.

2 Tous les documents saisis sur sa personne figurent dans son dossier déjà cité. Son arrestation fut annoncée par le Courrier de l'Ain, article reproduit dans le Journal de Genève du 12 mai 1871.

3 J. Guillaume, ibid.

4 Louis Andrieux, La Commune à Lyon en 1870 et 1871, Paris 1906, pp. 254-255. Paul Boegner, qui était alors chef de cabinet du préfet, parle d'une demi-douzaine d'individus arrêtés à leur descente du train, à Lyon, dont A. Leblanc, ce qui, pour ce dernier en tout cas, est manifestement faux. "L.yon en février-juin 1871", in: Revue de 1'Université de Lyon, 1929, pp. 280-281.

5 Observations de James Guillaume sur un manuscrit de Fritz Brupbacher (1911?), papiers Brupbacher, I.I.S.G. Amsterdam; Max Nettlau, "Nachträge", note 4467 , I.I.S.G.

${ }^{6} \mathrm{Cf}$. notamment le document II. 
Colonna et Perret parlent tous deux d'une colonne de volontaires prêts à partir de Genève pour fomenter la révolution en France. Plusieurs rapports de police y font allusion, mais sans grande précision. Nous ne disposons, à ce sujet, que d'un témoignage indirect, postérieur de onze ans aux événements. En 1882, J. Ph. Becker raconta à Reinhold Rüegg qu'on lui avait demandé, à la fin d'avril 1871, de former une troupe qui devait pénétrer en France pour y soulever les villes du Midi. Un fonds «relativement important» avait été mis à sa disposition. Il alla aussitôt demander conseil à l'un de ses amis, Guillaume Rüstow, ancien officier prussien, démocrate réfugié en Suisse après 1848; fixé à Zurich, Rüstow s'était fait naturaliser, était devenu colonel d'état-major et enseignait les sciences militaires. ${ }^{1}$ Mais l'échec du mouvement lyonnais puis l'entrée des Versaillais à Paris rendirent tous les plans sans objet. ${ }^{2}$

Relevons que ce projet n'était peut-être pas si irréalisable ou aberrant qu'on pourrait le croire aujourd'hui. Pour Becker, pour les Genevois et les Lyonnais, il s'inscrivait dans toute une série de mouvements analogues dont le souvenir était resté très vivace: 1831, première expédition de Savoie, depuis Lyon; 1834, deuxième expédition, dirigée par Mazzini, depuis Genève; 1848, expédition des Voraces, depuis Lyon, toujours contre la Savoie; 1848-1849, formation de corpsfrancs et soutien des insurgés badois depuis le territoire suisse $(\mathrm{J}$. Ph. Becker y fut tout particulièrement impliqué); 1851, tentative de quelques républicains français, au lendemain du coup d'Etat, pour franchir la frontière en bande armée, dans la région de Seyssel; aide aux patriotes italiens en 1848, en 1859-1860 .... Ajoutons qu'à Genève, outre les réfugiés de Lyon et du reste de la province, de nombreux volontaires garibaldiens avaient afflué; souvent mal vus et en butte aux tracasse-

1 Sur ce personnage, cf. Marcel Herwegh, Guillaume Rüstow. Un grand soldat, un grand caractère (1821-1878), Paris 1935, et, plus récent, Peter Wiede, Wilhelm Rüstow (1821 bis 1878). Ein Militärschriftsteller der deutschen Linken, Diss. Munich 1957.

" Reinhold Rüegg, "Aus Briefen an Johann Philipp Becker», in: Die Neue Zeit, VI (1888), p. 565; Becker avait promis à Rüegg de lui remettre tous les papiers relatifs à cet épisode; malheureusement, il l'oublia et, après sa mort, Rüegg fouilla vainement les archives du vétéran. Le fonds Becker, à l'I.I.S.G., ne contient rien à ce sujet, sinon deux télégrammes de Rüstow annonçant à Becker son retour à Zurich; ils sont datés du 25 avril et du 11 mai 1871. Dans les papiers Herwegh et Rüstow conservés au Dichtermuseum de Liestal, nous n'avons trouvé aucun élément nouveau, sinon la confirmation de déplacements de Rüstow: antérieurement au 22 avril, il s'était rendu en Suisse romande pour y placer ses enfants. Du 18 juin au 4 juillet 1871, il séjourna à Paris. Le 22 avril 1871, il écrivait à Emma Herwegh: "Die Herren der Pariser Kommune hatten mit ihrem Kravall auch wohl warten können, bis der noch vor zwei Monaten so grosse finanzielle Credit Frankreichs ausgenützt war, um den Prussiens ihr Lösegeld zu bezahlen und Frankreich von ihnen zu befreien.» 
ries des autorités françaises, ils avaient préféré franchir la frontière et constituaient une base de recrutement assurée.

Pour gagner le Sud-Est, les envoyés de la Commune de Paris avaient avantage à passer par Bâle, après avoir traversé le territoire occupé par les Prussiens; de là, par Genève, ils pouvaient rallier sans trop de difficultés Lyon ou d'autres villes de la région. C'est l'itinéraire qu'empruntèrent sans doute tous ceux qui réussirent à gagner les départements du Sud-Est et du Midi.

A la fin du mois d'avril, la Commune chargea Claude François Marchand de porter à Berne une Adresse de la Commune à la Suisse. Républicain de 1848 réfugié en Suisse, Marchand y avait de nombreuses relations, surtout dans la capitale fédérale où il tenait un commerce de vins. Profitant du fait qu'il connaissait personnellement le président de la Confédération, il n'eut aucune peine à se faire recevoir par lui, le $1^{\text {er }}$ mai, et à lui transmettre l'adresse dont il était chargé. En sortant du bureau, il croisa le ministre de France à Berne qui le reconnut, ce qui donna lieu à un léger incident diplomatique et à quelques polémiques dans la presse. ${ }^{1}$ Marchand ne se borna pas à cette démarche mais s'efforça de faire de la propagande en faveur de la Commune auprès de ses relations, dans les milieux radicaux qu'il connaissait bien et dans la presse. Plusieurs journaux reproduisirent l'adresse. ${ }^{2}$

Peu après, Artus revint en Suisse, muni cette fois d'une délégation en bonne et due forme, signée de Paschal Grousset en date du 6 mai:

«Un Comité d'action, chargé d'éclairer le Midi de la France sur la situation de Paris et sur le sens véritable de la révolution du 18 mars, est établi à Genève.

Le citoyen Artus en a la délégation et la direction de par la Commune de Paris (Co des Relations extérieures). Il a le droit de nommer des sous-délégués qui devront s'aboucher avec les représentants que la Commune envoie dans les différentes localités du Midi. Ces représentants recevront l'ordre en partant de Paris de correspondre avec le comité de Genève.» ${ }^{3}$

Nous ne savons pas quelles furent les activités du Comité d'action durant les quelque deux semaines qui précédèrent l'entrée des Versaillais dans la capitale. A une date indéterminée, Artus semble s'être

1 Dictionnaire biographique du mouvement ouvrier français, t. VII, Paris 1970.

2 La Suisse radicale, 1 er et 2 mai 1871, par exemple. A Paris, l'Adresse parut entre autres dans le journal de Lissagaray, le Tribun du peuple du 18 mai 1871. Le texte, pompeux et grandiloquent, était daté du 22 avril et signé des délégués à la Commission exécutive Viard, Paschal Grousset, Jules Andrieu.

3 M. Vuilleumier, "L'Internationale à Genève...", art. cit., p. 637. Le document est conservé à la Bibliothèque publique et universitaire de Genève (D. O.). 
rendu à Londres où il fit la connaissance d'Engels. ${ }^{1}$ Quelques rares indices laissent supposer que l'action se poursuivait; ainsi, le 12 mai, à Bellegarde, on découvrit, sur un typographe lyonnais qui venait de passer deux jours à Genève, Joseph Clément, un numéro de l'Egalité reproduisant l'une des adresses des sections genevoises en faveur de la Commune et diverses commandes signées de John Benoit, le directeur de l'Imprimerie coopérative qui avait prêté son passeport à Albert Leblanc. ${ }^{2}$ Faut-il voir en Clément un émissaire du comité ou un courtier d'imprimerie un peu imprudent?

Durant le mois de mai, ce sont les Lyonnais liés à Albert Richard et à l'Alliance qui semblent avoir repris l'initiative. "Tant que la Commune de Paris restait debout, écrira J. Guillaume, on pouvait espérer encore et un groupe de Lyonnais organisa un <Comité central républicain socialiste de la France méridionale> qui fit imprimer à Genève diverses proclamations affichées dans le courant de mai et dans plusieurs autres villes.) $)^{3}$ Un jeune tisseur lyonnais, Claude Lance, fut arrêté à Bellegarde, le $20 \mathrm{mai}$, et trouvé porteur d'épreuves corrigées d'affiches signées de ce comité ainsi que d'une Protestation des républicains socialistes contre les dernières élections municipales. On trouva encore sur lui des lettres adressées à Dumartheray, Perron et Ozeroff. ${ }^{4}$ On peut supposer que l'action encore mal connue de Victor Cyrille à Lyon, à la même époque, est à mettre en rapport avec ce comité. Le 27 mai, en effet, des affiches multigraphiées, signées "Victor Cyrille, correspondant de Paris», furent apposées sur les murs de Lyon; il s'agissait d'une Proclamation à l'armée et d'un Appel à la province. Le 30 mai encore, d'autres affiches furent apposées; l'une, imprimée, était un appel «Au peuple» pour secourir Paris à l'agonie et était estampillée d'un timbre humide rouge: "Commune de Paris. Comité d'action des provinces». 5 Mais, comme l'écrira encore J. Guillaume, "la propagande du Comité

1 C'est ce qui ressort de la lettre d'Artus à Engels, datée de Paris, le 28 mai 1883: "Mon cher Général, il y a 12 ans que nous ne nous sommes vus.... Si mon souvenir n'est pas effacé de ta mémoire, donne-moi de tes nouvelles." Fonds Marx-Engels L 110, I.I.S.G.

2 A.D.R., M, Correspondance De Gourlet, Bellegarde, 15 mai 1871.

3 J. Guillaume, op. cit., t. II, p. 148.

4 A.D.R., 4 M 6, 3, copie de la main d'Oscar Testut. C'est sans doute là que fut découverte la lettre de Gaspard Blanc et d'Albert Richard à Charles Perron, relative au transport d'un ballot d'imprimés de Genève à Annecy et Seyssel, publiée par Testut.

5 Archives Nationales Paris, $\mathrm{BB}^{30} 486$. Notre hypothèse se fonde essentiellement sur l'action ultérieure de Victor Cyrille, en 1872-1873, où il sera l'un des éléments les plus remuants du groupe de Bakounine. On lit, dans le Journal officiel de la Commune du 17 mai: "Une délégation émanant de la Commune de Paris est en train de fonctionner à Lyon. Elle ne tardera pas à donner d'utiles résultats." 
central de la France méridionale ne produisit aucun résultat. A partir de la fin de mai, Albert Richard et Gaspard Blanc disparurent de la scène. . ${ }^{1}$

De leur côté, les militants jurassiens étaient en relations avec Besançon, où une section de l'Internationale s'était créée, au début de 1870 , et avait survécu, semble-t-il, à sa dissolution et à la condamnation de ses dirigeants, en juin $1870 .{ }^{2}$ "J'ai quelques raisons de croire que des publications venant de Neuchâtel et prêchant l'adhésion à la Commune ont circulé dans les ateliers», déclarait un maître tailleur, devant le juge d'instruction, en juillet $1871 .^{3}$ A deux reprises, des proclamations en faveur de la Commune furent affichées. ${ }^{4}$ En mai, les Neuchâtelois avaient élaboré un plan que nous dévoile Guillaume: «Des internationaux de nos diverses sections, armés, auraient passé la frontière en trois ou quatre groupes, se dirigeant vers cette ville où leur arrivée aurait coïncidé avec un soulèvement de la population ouvrière.» Un délégué était venu de Besançon et l'assemblée générale des sections jurassiennes, tenue à Neuchâtel, le 21 mai, avait désigné J. Guillaume et Treyvaud pour aller organiser le soulèvement sur place. ${ }^{5} \mathrm{Au}$ dire de Nettlau qui, avant la publication des souvenirs de Guillaume, avait mené sa propre enquête, ce dernier était très pessimiste à l'idée d'entreprendre ces opérations militaires pour lesquelles il n'avait aucune sorte d'expérience. ${ }^{6}$ Ajoutons que le pessimisme de Guillaume était certainement dû à son analyse de la situation. Ne partageant pas les illusions putschistes de beaucoup des partisans de Bakounine, connaissant l'état d'esprit de la province française et, en particulier, de la région frontalière, prévoyant déjà la chute de la Commune, il ne fut sans doute jamais intimement convaincu de la justesse de ce projet.

Ainsi, l'Internationale en Suisse et les réfugiés de la province ont pris une part beaucoup plus active qu'on ne pourrait le croire à la lutte de la Commune; ils l'ont fait non seulement par la propagande, par le

1 J. Guillaume, ibid.

2 La fondation de la section fut annoncée à la séance du Conseil général du 1 er mars 1870 (The General Council of the First International 1868-1870, Minutes, p. 214). Le mémoire inédit de Monique Boccard-Gavillon, L'Internationale à Besançon (1868-1871), Besançon 1963, 29 pp. dact., ne fournit aucune indication sur les relations avec les internationaux de Suisse.

3 Archives Nationales, C 2882, rapport du procureur, 20 juillet 1871 . Ce dernier ne pensait pas qu'il existait alors une section de l'A.I.'T. à Besançon, bien que certains jeunes tailleurs pussent y être favorables.

4 Ibid., C 2885, rapport du préfet du Doubs, 14 juillet 1871.

$5 \mathrm{~J}$. Guillaume, t. II, p. 152. Il ne nomme pas la ville, mais tout indique qu'il s'agit bien de Besançon, comme l'indique d'ailleurs Nettlau, "Nachträge», note 4467, I.I.S.G.

6 M. Nettlau, ibid. 
vote de résolutions, dans les réunions publiques, mais encore par des actions concrètes: envoi d'émissaires, établissement de contacts, acheminement de matériel de propagande, préparation d'une incursion armée. P. Lavrov, qui fut envoyé comme émissaire à Bruxelles et à Londres, estimait que la Commune avait fait beaucoup trop peu pour organiser des relations suivies avec la province et avec le mouvement ouvrier des pays voisins; il y voyait la conséquence de l'ignorance systématique, de la part des Parisiens, de tout ce qui se fait en dehors de leur capitale. ${ }^{1} \mathrm{Si}$, en gros, son jugement demeure valable, il mérite néanmoins d'être quelque peu nuancé.

\section{Proscrits}

A l'étranger, les proscrits vont se trouver confrontés à un problème délicat: celui de leurs rapports avec la population locale. En Angleterre, les difficultés de langue rendirent plus difficile l'adaptation. Mais en Belgique et en Suisse romande, où cet obstacle n'existait pas, l'intégration des réfugiés dans leur nouveau milieu n'en fut pas beaucoup plus aisée: la mentalité des uns et des autres était par trop différente. Rien ne le montre mieux que les heurts qui se manifestèrent au sein des sections de l'Internationale à Genève et sur lesquelles les lettres de Perret et d'Outine d'une part, de Malon et de Lefrançais de l'autre, nous fournissent nombre d'informations et portent des jugements, certainement injustes et déformés par la passion, mais bien significatifs.

Pourtant, les militants genevois avaient accueilli favorablement les proscrits; ils avaient tout fait pour faciliter leur fuite et lutté énergiquement pour leur assurer un asile. ${ }^{2}$ Une partie des réfugiés avait appartenu, en France, à l'Internationale; d'autres ne tardèrent pas à y adhérer dès leur arrivée en Suisse. Pourtant, malgré l'internationalisme dont chacun se réclamait, les incompréhensions et les frictions entre les deux groupes nationaux ne tardèrent pas à se manifester. On les assimile généralement au conflit qui déchirait l'Internationale et l'on sait qu'en effet la plupart des communeux réfugiés en Suisse se rangèrent aux côtés de Bakounine et de ses amis. Cela tient pour une part aux relations qui s'étaient nouées, avant la guerre déjà, entre les «alliancistes» suisses et un certain nombre de militants français. Ceux-ci, réfugiés en Suisse, prirent tout naturellement parti pour leurs amis. Mais le choix de la masse n'était pas déterminé uniquement par les relations de quelques individus, fussent-ils des plus influents. Il ne provenait pas

1 P. I. Lavrov, Die Pariser Kommune vom 18. März 1871, Berlin 1971, p. 131. 2 M. Vuilleumier, "Le gouvernement de Versailles, les autorités suisses et les proscrits de la Commune», in: Le Mouvement social, no 28 (janvier-mars 1962), pp. 28-46. 
non plus d'une analyse purement rationnelle des théories politiques qui s'opposaient; celles-ci étaient d'ailleurs à tel point masquées par les conflits de personnes et d'organisations, par les circonstances purement locales, que rares étaient sans doute les militants capables de comprendre toutes les implications de la lutte et d'en dégager l'aspect théorique.

En fait, le choix des communeux répond à une double réaction de leur part. Pour ces hommes, plongés depuis 1868 dans la vie politique intense de Paris, ayant vécu toutes les péripéties de la guerre, du siège et de deux révolutions, combien pâle et inintéressante devait être la vie de l'Internationale à Genève. Sections professionnelles en quasi léthargie, presque entièrement absorbées par le train-train quotidien et les affaires du métier; section centrale dominée par des préoccupations de politique locale, où les éléments les plus modérés donnaient le ton et se souciaient avant tout de ne pas compromettre, par des excès de langage ou des actes de violence, leur alliance électorale avec les radicaux, tout cela n'avait rien de bien attrayant pour les communeux et les rendra particulièrement réceptifs aux critiques formulées par les "alliancistes» locaux. Ayant vécu dans un milieu tout différent, participant de traditions culturelles et historiques tout autres, enrichis d'expériences sans commune mesure avec celles des Genevois, les réfugiés, surtout les Parisiens, étaient totalement "déphasés» par rapport au mouvement ouvrier local. ${ }^{1}$

Aussi, après avoir tenté de modifier l'orientation des sections genevoises, la plupart d'entre eux délaissèrent-ils l'organisation locale pour se consacrer aux activités de la Section de propagande, membre de la Fédération jurassienne, dont toute l'action se voulait dirigée vers la France. Les seuls à demeurer au sein de l'organisation genevoise furent un certain nombre d'ouvriers qui s'intégrèrent au sein de leurs associations professionnelles respectives: Le Péhive, Tacussel, Lauprêtre, Guittat, Bazin, Piéron... L'adhésion de Cluseret, au début de 1872, à son arrivée à Genève, ses prises de position en faveur du Conseil général répondaient sans doute à d'autres motifs: honni par nombre de communeux, il trouvait profitable de s'allier à leurs adversaires, espérant peut-être jouer ainsi un rôle au sein de l'organisation. ${ }^{2}$

Mêlée à cette réaction due à la différence des expériences, s'ajoutait l'incompatibilité des nationalismes. En effet, l'internationalisme avoué des uns et des autres recouvrait, en fait, des sentiments nationaux bien enracinés. Chez les communeux, l'héritage du jacobinisme se manifeste par la persistance d'une croyance solidement implantée chez les

1 Cf. document $\mathrm{V}$.

2 M. Vuilleumier, "Les proscrits de la Commune en Suisse (1871)», in: Revue suisse d'histoire, XII (1962), pp. 498-536. D'autres témoignages confirment le jugement de Malon et Lefrançais. 
révolutionnaires: celle d'appartenir à la nation la plus avancée du monde, appelée à montrer la voie de l'affranchissement humain sinon à libérer elle-même les autres peuples. Ce messianisme révolutionnaire sort encore accru de la défaite de la Commune et l'adhésion à celle-ci des socialistes étrangers ne fera que le renforcer. Nul ne l'a mieux exprimé que l'observateur fin et perspicace que savait être Arthur Arnould, et son texte doit être largement cité:

"Lorsqu'on parle de peuple, une fois les frontières de la France passées, il ne faut plus croire qu'il s'agisse de ce grand peuple fort et doux, intelligent et fier, - peuple volcan d'où jaillit l'avenir, au bruit de la foudre, à la lumière des éclairs, - creuset immense et bouillonnant où s'élabore le progrès, - glaive, force, salut de la France, comme il sera le salut de l'Europe.

A Genève, entouré de tous côtés par la France, à Genève, de langue française, il y a encore une sorte de remous, comme sur les côtes de l'Océan.

Après Genève, et à mesure que vous vous éloignez, cela diminue à chaque pas en avant. Le peuple redevient troupeau, troupeau qui regimbe plus ou moins contre le berger, qui bêle plus ou moins sous le couteau. C'est la foule qui souffre et se plaint, et qui rêve au meilleur sort, foule qui a ses vertus et ses mérites, ses courages et ses vaillances, qui fait effort pour se relever et qui entrevoit déjà l'aurore éclatante de la justice, - mais garrottée encore des langes du passé, patiente, résignée, dont le cerveau n'est pas affranchi, dont la conscience est confuse, et qui garde au fond du cœur le respect de la force.

Mille préjugés, préjugés religieux, préjugés de race et de clocher, alourdissent son pas, bouchent les avenues de sa pensée. La rupture avec hier n'est pas complète, absolue, raisonnée. Elle commence seulement. [...] L'amour de l'humanité ne se trouve que dans la phraséologie révolutionnaire, empruntée à nous, - où les mots de fraternité, de solidarité, d'égalité, de droit, n'ont plus la même portée, parce que ce sont des mots appris. Ils ne sont pas sortis tout armés des entrailles mêmes des peuples qui les balbutient aujourd'hui.

Cela est triste, mais cela est vrai : - Oui, il n'y a qu'un seul soldat de l'humanité - la France!

Pourquoi, parce que la France est la seule nation qui ait le sentiment profond, vivace, instinctif de la solidarité universelle.

Parce que Paris, qui est en avance de vingt ans sur le reste de la France, - et qui le paye - est en avance d'un siècle sur le reste de l' Europe. 
Je le dis non par chauvinisme - j'appartiens à un parti qu'on accuse de tout, sauf de cela - je le dis parce que je le crois, et je le crois parce que je l'ai vu. [...]

Grattez le Russe, dit-on, et vous trouverez le Cosaque. Grattez le Suisse, l'Italien, l'Espagnol, l'Allemand, l'Anglais, l'Américain, vous trouverez le Suisse, l'Italien, l'Espagnol, l'Allemand, l'Anglais, l'Américain.

Grattez le Français, vous trouverez l'homme.

[...] Certes, le souffle démocratique a répandu dans le monde des germes, qui poussent, se développent, quoi qu'on fasse, quoi qu'il arrive; mais si cette grande lumière qui rayonne de Paris s'éteignait, le chaos recommencerait.

Il n'est, d'ailleurs, pas à désirer que ces différences de tempérament disparaissent en un jour. Chaque peuple apporte au grand travail de l'humanité, grosse du progrès, des éléments spéciaux de succès, des aptitudes personnelles, dont le concours et la collaboration sont essentiels à la victoire définitive du droit sur la force, de la justice sur le privilège, de la solidarité sur la guerre. Quelques-uns de ces peuples ont des qualités pratiques qui nous manquent parfois, quand, dans nos grands coups d'aile, nous tentons d'arriver jusqu'à l'extrême frontière de notre idéal....

Seulement, il faut que la France qui, dans ses larges conceptions, travaille pour tous, sache bien quelle est sa situation vraie, et ne se fasse pas d'illusions sur l'esprit du milieu dans lequel et pour lequel elle agit. Il faut qu'elle sache nettement que seule, aujourd'hui encore, elle a le sentiment désintéressé, non pas seulement de la fraternité, mais aussi de la solidarité et qu'elle doit compter sur elle-même, mais sur elle exclusivement....

[...] Elle est bien vraiment la fille aînée de l'humanité. A elle de donner l'exemple. Qui a plus, doit plus. Elle a un rôle à jouer que nul ne peut jouer. Elle occupe une place que nul ne peut occuper. Son peuple porte au front une couronne, - couronne d'épines, que nul ne pourrait ceindre s'il la laissait jamais glisser de son front ensanglanté. Il a charge d'affranchissement. Il n'a pas le droit d'abdiquer, ni même de se lasser. Ce serait la plus grande trahison de l'histoire. Mais il ne doit compter, pour longtemps encore, en fait sinon en paroles, que sur l'ingratitude, et il n'a point à s'endormir dans une fausse sécurité....

$[\ldots]$ Ceux qui nous doivent trop ne nous pardonnent que le jour où ils valent autant que nous. Ce jour n'est pas levé.»1 
Texte admirable où s'exprime avec candeur un sentiment largement répandu, mais plus maladroitement exprimé chez d'autres. Deux ans plus tard, en 1876, on lit dans un texte de Bastelica: "La France est un Etat, mais avant tout elle est une humanité, écrivais-je en 1868, et je le soutiens. Cette humanité, qui recèle dans son sein tous les progrès accomplis depuis 18 siècles, que l'on voudrait peut-être détruire ou tout au moins annihiler», c'est le devoir de tous les Français de la défendre contre le pangermanisme. ${ }^{1}$ Cette assimilation de la France à l'humanité, cette croyance inébranlable dans sa mission révolutionnaire et régénératrice étaient encore accrues par l'amertume de l'exil, le mal du pays et les difficultés réelles à s'adapter aux mœurs de l'étranger. Cette nostalgie, les lettres de Clémence la traduisent d'une manière touchante. Lui qui aimait son métier de relieur, il n'avait pu le reprendre et, après diverses tentatives infructueuses, avait dû exercer la profession de comptable. D'où son chauvinisme... en matière de reliure! De plus, ce militant, ami et compagnon d'armes de Varlin, qui avait participé à toutes les luttes de la fin de l'Empire, était exilé dans une petite bourgade provinciale où, en fait, le mouvement ouvrier était inexistant. L'Internationale y était en voie de disparition et les efforts de la Fédération jurassienne pour y faire vivre une petite section ne réussirent guère à regrouper plus qu'une poignée d'adhérents. ${ }^{2}$

Le patriotisme et le messianisme jacobin des Français se heurtaient au sentiment national des internationaux suisses, et cette opposition ne faisait que raidir un peu plus chacun des antagonistes dans ses attitudes. Cela d'autant plus que, dès ses débuts, on pourrait presque dire sa création, l'idéologie nationale helvétique a eu, elle aussi, son aspect messianique: la Suisse avait une mission, celle d'enseigner la liberté et sa pratique aux peuples qui l'avaient perdue et oubliée. Le succès des mouvements libéraux dans les différents cantons, après 1830, l'issue

1 André Bastelica, Appel aux Français. Lettre d'un proscrit sur la situation, Genève $1876,2 \mathrm{pp}$. fol.

2 Ces lettres sont conservées à 1'I.I.S.G., Collection Descaves. Elles sont très révélatrices de la personnalité sympathique de leur auteur. Voici le passage de l'une d'entre elles, adressée de Lausanne, le 24 septembre 1883, à Wynants, membre comme lui de la Société des Relieurs de Paris: "Merci encore de votre bonne surprise et de la charmante reliure qui enveloppe si bien l'œuvre de votre pensée.... Si vous saviez combien il m'est agréable de voir, toucher, regarder et analyser une reliure parisienne. Cela m'arrive si rarement et ce que l'on fait ici est si mastoque, si mal raisonné, si allemand en un mot que c'est à vous dégoûter des reliures et surtout des relieurs. Je ne suis pourtant pas chauvin, moi, un des fondateurs de l'Inter[nationale] mais le goût allemand, le commerce allemand, l'exploitation allemande vous doutez-vous de ce que c'est, ô bonasses vaincus parisiens!" 
heureuse de la crise du Sonderbund et le maintien d'une Suisse libérale au sein d'une Europe livrée à la réaction après 1849 n'avaient fait qu'accroître ce sentiment d'une Confédération gardienne de la liberté des peuples. La constitution d'Etats nationaux fondés sur la communauté linguistique, qui menaçait l'existence même de cette Confédération, provoqua une extension de la mission attribuée à la Suisse; ce n'était plus seulement conserver les libertés mais encore unir des peuples différents en un Etat d'une forme supérieure aux Etats nationaux, être en quelque sorte le prototype d'une Europe nouvelle, celle des peuples fraternellement unis. Et l'écroulement du second Empire semblait justifier rétrospectivement cette idée; n'avait-il pas constitué la principale menace pour l'indépendance, l'intégrité et les libertés de la Suisse? Dernière composante de ce sentiment messianique: l'élément caritatif. La fondation de la Croix-Rouge, en 1864, les secours apportés aux blessés de la guerre de 1870-1871 et aux rescapés de l'armée Bourbaki en sont les premières manifestations justificatrices. La Suisse, première république du continent, gardienne des libertés démocratiques; la Suisse symbole de la compréhension entre les peuples et prototype d'une Europe nouvelle; la Suisse participant à la souffrance des autres par son action charitable, telles sont, en 1871, les trois composantes d'un sentiment national largement répandu et unanimement accepté. ${ }^{1}$

Mais cette analyse ne doit pas nous faire oublier qu'en Suisse le patriotisme a une dominante essentiellement locale, qu'il se fonde en tout premier lieu sur l'appartenance à une ville, à un canton, que l'on est, surtout, à cette époque, genevois ou fribourgeois avant d'être suisse. Le sentiment national, dont nous avons dit les composantes, a quelque chose d'artificiel; originairement, il a été créé de toutes pièces par les intellectuels de la fin du XVIIIe siècle qui l'ont conçu et utilisé comme un moyen d'intégration des masses au nouvel Etat issu des bouleversements révolutionnaires. Au contraire, le patriotisme local a quelque chose de spontané, de naturel. Qu'il y ait eu contradiction entre les deux, en certains endroits, à certains moments, c'est bien évident. La crise du Sonderbund et ses suites en sont la meilleure illustration. Mais dans les milieux radicaux et libéraux, dans les cantons avancés, la synthèse s'est assez harmonieusement réalisée.

C'est le cas pour Genève, où toute la tradition républicaine et

1 Sur ces questions encore mal étudiées, cf. Daniel Frei, Die Förderung des schweizerischen Nationalbewusstseins nach dem Zusammenbruch der Alten Eidgenossenschaft 1798, Zurich 1964. Cette thèse reste évidemment centrée sur la fin du XVIIIe siècle, malgré quelques incursions très éclairantes sur le XIXe et le XXe siècle. 
réformée est capitalisée par ce sentiment patriotique local qui se veut national et, pour cela, s'intègre les éléments analysés plus haut. Cela n'empêche pas, d'ailleurs, à l'intérieur même de cette tradition, l'apparition de clivages. C'est ainsi que les internationaux genevois qui, en accord avec une fraction du parti radical, présentaient deux candidats lors de l'élection du gouvernement cantonal, en novembre 1871, se réclamaient, dans leur manifeste, non seulement de la glorieuse et populaire révolution radicale de 1846 , mais encore des premiers artisans de l'indépendance genevoise et de l'alliance avec la Suisse, au $\mathrm{XVIe}$ siècle; ils en faisaient le départ d'une tradition anti-calviniste, qu'ils opposaient à celle dont se réclamaient les conservateurs protestants: "Le parti populaire et ouvrier fait dans ce sens un appel chaleureux à tous les radicaux qui ne veulent pas déserter le drapeau de 1846, aux libres-penseurs, aux travailleurs de la ville et de la campagne. Il fait appel à tous nos confédérés des autres cantons qui peuvent certifier à la face de la Suisse et de l'Europe que le parti populaire est fermement attaché à la patrie suisse, dont il a le premier recherché l'alliance. Nos ancêtres étaient les Eidguenots et les Libertins, et non les Mameluks et les Calvinistes français. $\rangle^{1}$

Ce patriotisme local, partie intégrante du sentiment national, n'est remis en cause par personne et on y adhère d'autant plus bruyamment qu'on se veut plus radical et d'idées politiques plus avancées. Aussi se retrouve-t-il chez tous les Genevois membres de l'Internationale. Ceux-ci avaient soutenu la Commune, mais en voyant en elle en somme une continuation des révolutions genevoises de 1841 et 1846 , qui avaient renversé l'ancienne aristocratie protestante et donné le pouvoir à la bourgeoisie radicale, et l'Egalité avait écrit: «Ce que Paris veut, ce que les autres villes républicaines de la France demandent, en acclamant la Commune, ce n'est guère plus que ce que les cantons suisses possèdent. $\eta^{2}$ La conclusion logique, le même journal la tirait au moment où succombait la Commune:

«Dans un pays comme la Suisse, où les institutions républicaines, depuis longtemps conquises, assurent au citoyen certaines garanties de liberté, dites publiques, on n'a plus besoin de combattre à main armée les attentats de la réaction monarchique; le peuple

1 Journal de Genève, 12 novembre 1871, et autres journaux genevois. Entre 1515 et 1520 , les Eidguenots étaient les Genevois partisans d'une alliance avec les cantons suisses pour émanciper leur ville de la suzeraineté du duc de Savoie; par dérision, les partisans de ce dernier avaient reçu le surnom de "Mamelus» ou "Mamelouks». Les «libertins» représentaient le parti des Genevois réformés, opposés à la discipline et aux lois imposées par Calvin.

2 L'Egalité, 31 mars 1871. 
travailleur n'a qu'à poursuivre avec énergie son regroupement, basé sur les principes socialistes, pour parvenir ainsi par l'organisation ouvrière, étendue sur toute la contrée, à la direction souveraine des affaires du pays; c'est-à-dire que le peuple, en Suisse, peut arriver paisiblement au triomphe de la représentation du travail par les moyens que les institutions républicaines lui offrent, à moins que la réaction ne veuille lui suggérer [sic] des entraves, en s'insurgeant contre les droits républicains du peuple et en cherchant à supprimer le mouvement ouvrier..1 ${ }^{1}$

Cet évolutionnisme n'était peut-être pas partagé par tous les internationaux genevois (J. Ph. Becker, par exemple), il n'en était pas moins accepté sans protestation. Il correspondait bien à des sentiments largement répandus dans les masses, et, justement, le mouvement ouvrier genevois, à cette époque, avait incontestablement un certain caractère de masse que l'on ne retrouvait pas dans le reste du pays.

On conçoit facilement l'irritation que les communeux devaient ressentir devant le nationalisme moralisateur des Suisses et inversement la colère que provoquait, chez ces derniers, le nationalisme jacobin des premiers. Les témoignages de ce heurt sont nombreux et on pourrait en multiplier les exemples. Cette opposition de deux mentalités se manifeste également par toute une série d'incidents au niveau de la vie quotidienne, des relations personnelles. Elle explique le repliement des proscrits sur eux-mêmes, le peu d'influence qu'ils eurent sur le mouvement ouvrier suisse et le peu d'éléments positifs qu'ils retirèrent de leur expérience du pays.

Tous cependant n'eurent pas cette attitude. D'abord parce que la Fédération jurassienne se montra sensiblement plus accueillante que les sections genevoises; à Genève, les proscrits constituaient la grande majorité de sa section; dans le Jura, peu nombreux, ils s'intégrèrent sans grande difficulté dans les petites sections locales, beaucoup plus facilement précisément qu'à Genève où leur nombre même était déjà un obstacle à une telle intégration: un ou deux individus isolés trouvent plus facilement leur place dans un petit groupe que plusieurs dizaines de personnes au sein d'une organisation d'une certaine importance, avec ses habitudes, ses traditions. Enfin, les idées défendues par la Fédération jurassienne correspondaient sûrement mieux aux aspirations des communeux que les pratiques électoralistes d'une partie des dirigeants genevois.

Néanmoins, comme nous l'avons vu, un certain nombre de proscrits réussirent à s'intégrer au mouvement ouvrier local où ils jouèrent un 
certain rôle. Retenons le cas de Gustave Bazin, qui fut l'un des dirigeants de la grève des ouvriers bijoutiers de Genève, en 1872-1873, et secrétaire du Comité fédéral régional suisse de l'A.I.T. Passé en Belgique, il tint encore une certaine place dans les organisations ouvrières de Bruxelles avant de se fixer à Londres, en 1877, où on le retrouve dans les réunions socialistes internationales au Duke of York. ${ }^{1}$ Citons encore Arthur Piéron, typographe, qui, travaillant de son métier à Lausanne, donna toute une série d'études au Gutenberg, l'organe des ouvriers typographes de la Suisse romande. En 1873, on le retrouve à Rome où il s'attaque aux tendances centralisatrices et autoritaires du Comité central de la Société typographique italienne, de tendance mazzinienne. Revenu à Lausanne, il se fixa, en 1875, à Genève où il joua un rôle important, non seulement au sein de son syndicat, mais aussi dans le parti socialiste naissant. Collaborateur du Précurseur, petit journal que publiait J. Ph. Becker et qui s'efforçait de jeter les bases d'un tel parti, orateur habituel des réunions socialistes, Piéron fut, jusqu'en 1880 , l'un des principaux militants du mouvement ouvrier à Genève. On le retrouvera à Paris, dans les rangs possibilistes, puis allemanistes. ${ }^{2}$

Bien entendu, l'étude de la proscription communaliste en Suisse ne peut se limiter à ces deux problèmes: le soutien des réfugiés de la province et des internationaux suisses à la Commune de Paris; les difficultés dues aux différences de mentalités, d'expériences vécues et à l'opposition des nationalismes. Mais il ne peut être question d'examiner en détail toutes les questions soulevées par une étude qui se voudrait exhaustive. Bornons-nous, pour éclairer la lecture des documents publiés ci-après, à relever encore la violence des conflits qui opposaient les réfugiés entre eux. Si l'exil, par la coupure qu'il provoque (et les loisirs involontaires qu'il procure), favorise la réflexion politique et la décantation des expériences antérieures, il tend aussi à cristalliser images et théories, à les rendre schématiques et abstraites. Par le divorce qu'il entraîne entre les émigrés et leur milieu naturel, il empêche ceux-ci de suivre l'évolution de leur pays, il tend à en faire des gens qui perdent tout contact avec la réalité, celle de leur patrie aussi bien que celle du pays qui les accueille et auquel ils ne peuvent s'adapter.

D'où l'importance du passé, des vieilles querelles, d'où les innombrables clivages et les luttes de chapelles continuelles. D'où aussi la profusion des accusations souvent ignominieuses que se lancent les adver-

1 Dictionnaire biographique du mouvement ouvrier français, t. IV, Paris 1967. Sur l'activité de Bazin à Bruxelles, cf. l'article de D. E. Devreese publié cidessus, pp. 268 et 270 .

2 D.B.M.O.F., t. VIII, Paris 1970. 
saires. ${ }^{1}$ Outre celle de mouchard et de collaborateur occulte de tel ou tel pouvoir, l'une des plus fréquentes est celle de «bonapartiste». L'évolution d'Albert Richard et de ses amis' avait mis en garde les proscrits et beaucoup étaient prêts à détecter de telles tendances chez leurs ennemis. Or les républicains bourgeois ne se faisaient pas faute d'appliquer l'épithète aux socialistes, aux communeux et à tous ceux qu'ils accusaient de compromettre la République par la violence de leurs opinions et leurs revendications exagérées. Rien d'étonnant donc si les proscrits donnèrent aussi dans ce travers. Il suffisait de peu pour alimenter les soupçons, ainsi que nous l'explique la lettre de Jaclard à Marx, qui a également le mérite de nous montrer la façon dont Richard

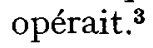

\section{DOCUMENTS}

\section{I-IV: LETTRES DE HENRI PERRET A HERMANN JUNG}

I

\section{Particulière}

Genève, le 4 juin 1871

Cher ami Jung,

Voilà bien longtemps que nous n'avons correspondu ensemble; les événements qui se passent en France absorbent complètement toutes nos idées pour penser à autre chose. J'ai le cœur trop serré à la suite des événements de Paris pour pouvoir vous écrire une longue lettre, il y a trop de nos frères égorgés, et d'autres dont l'existence est en danger pour avoir la tête assez tranquille pour écrire; il faut autre chose que cela, c'est d'agir et c'est ce que nous faisons actuellement, nous sommes un petit groupe d'hommes décidés à tout, pour sauver les derniers survivants de la prise de Paris, nous veillons activement et nous agissons avec prudence mais sans perdre de temps. Pour aujourd'hui je ne puis vous donner d'autres détails, dans quelques jours et quand nous serons renseignés complète-

1 Cf. Oscar Blum, "Zur Psychologie der Emigration», in: Archiv für die Geschichte des Sozialismus und der Arbeiterbewegung, VII (1916), pp. 412-430. C'est une des seules études systématiques (trop systématique, sans doute). Malheureusement, l'auteur n'avait pas une connaissance suffisante des différentes émigrations et se réfère trop exclusivement à Passé et pensées de Herzen.

2 Cf. l'article et les documents publiés par M. Moissonnier ci-dessous.

3 Cf. document VII.

4 Dans sa correspondance avec Hermann Jung, secrétaire correspondant pour la Suisse du Conseil général, à Londres, Henri Perret, le secrétaire général de la Fédération romande, doublait sa lettre officielle d'une missive plus libre et plus personnelle portant la mention "particulière". Perret a un style et une orthographe déplorables. Pour en faciliter la lecture, nous nous sommes permis quelques retouches de ponctuation et d'orthographe, quand des graphies par trop fantaisistes rendaient la compréhension du texte particulièrement pénible. 
ment par nos envoyés, vous recevrez une longue lettre de tout ce que nous avons fait pour aider Paris.

Vous jugerez, cher ami, si vos amis de Genève ont bien travaillé pour la révolution sociale, que nous espérions voir réussir; je suis trop profondément affligé de tout ce qui se passe pour pouvoir vous écrire une lettre correcte, ma main tremble de colère en envoyant [sic] tout ce qui se passe en France.

Bonne nouvelle, notre chère sœur Elise est sauvée: elle a quitté Paris au milieu de toutes sortes d'obstacles et à travers les obus et les balles; c'est un miracle qu'elle soit sauvée, nous l'avons à Genève et nous la gardons précieusement; elle a pu s'échapper avec quelques amis et amies de cette terrible tuerie; elle doit vous écrire. ${ }^{1}$

Les amis vous saluent: Outine, Grosselin, Dupleix et Becker. ${ }^{2}$

A bientôt cher ami et je compte sur vous pour nos demandes au Conseil général.

Je vous serre la main fraternellement.

Fonds Jung 899, I.I.S.G.

H. Perret

Particulière

Genève, le 23 juillet 1871

Mon cher ami Jung,

Vous voudrez bien faire connaître au Conseil général notre demande et faire tout votre possible pour que nous ayons une réponse au plus vite. ${ }^{3}$ La citoyenne Elise m'a communiqué que vous lui aviez dit dans une lettre que bientôt je recevrais quelque chose; $\mathrm{j}$ 'attends toujours pour le Comité fédéral et pour moi. Vous me délaissez un peu depuis quelques temps; je sais que vous avez beaucoup à faire et que vous devez être parfois très fatigué, mais un petit bout de lettre de loin en loin me ferait plaisir.

Dans une de mes dernières lettres, je vous avais mentionné que je vous donnerais quelques détails sur ce que nous avions fait pendant que la Commune de Paris se battait contre Versailles. Dans une petite réunion où nous discutions sur ces événements et les moyens de travailler la province pour aider Paris, nous avons décidé de former un Comité secret. Il était composé de Becker, Outine,

1 Il s'agit d'Elisabeth Dmitrieff, qui, après avoir milité dans l'Internationale à Genève, en 1870, avait gagné Londres, où elle fréquenta la famille de Marx. Elle gagna Paris sous la Commune et joua un rôle de premier plan à l'Union des Femmes pour la défense de Paris et les soins aux blessés. En octobre 1871, elle rentra en Russie.

2 Grosselin, ouvrier horloger; l'un des dirigeants les plus populaires de l'Internationale à Genève. Plus radical que socialiste, il fut, à l'automne 1871, candidat aux élections du gouvernement cantonal. Jean-Baptiste Dupleix, ouvrier relieur français, réfugié à Genève à la suite du coup d'Etat de 1851, joua un rôle important dans les débuts de l'Internationale dont il fut, à Genève, l'un des fondateurs.

${ }^{3}$ Le Comité fédéral romand avait demandé s'il était exact, comme le prétendaient les dirigeants de la Section de l'Alliance internationale de la démocratie socialiste à Genève, que cette société avait été admise dans l'A.I.T. par le Conseil général. 
Troussoff, Duval, un membre du Comité fédéral, et moi. ${ }^{1}$ Nous avons écrit à quelques amis que nous avions en France, et aussi par l'intermédiaire de citoyens français [réfugiés?] à Genève, venant de St Etienne, Marseille et Lyon. Nous avons fait venir un brave citoyen de Lyon, homme parfaitement sûr, à qui nous avons confié nos idées et qui a approuvé nos plans. Nous avons fait un manifeste qui a été répandu dans la province. Il était censé être parti d'un Comité central fixé à Lyon. Nous avons eu une active correspondance avec divers groupes révolutionnaires de France. - Avec la présente vous recevrez diverses pièces émanant de notre Comité d'action - Notre ami Duval est allé à Lyon, un autre est allé à Marseille et à d'autres villes pour préparer le mouvement. Notre circulaire et une affiche ont été répandues à profusion dans diverses villes. Nous voulions aider à la formation de Comités d'action partout où il y avait des hommes résolus. Nous n'avons rien négligé, ni temps ni argent, pour faire quelque chose. Nos séances se sont prolongées souvent fort tard dans la nuit. Tout faisait supposer qu'il serait possible de soulever les villes principales de la province. Sur ces entrefaites arriva le citoyen Artus délégué par la Commune dans la province et à l'étranger. Il est de Genève. Grousset lui avait donné un mandat régulier. Nous lui avons fait part de nos travaux, il est reparti pour Paris; il a fait part à la Commune de notre plan et du Comité que nous avions formé; il est revenu avec plein pouvoir pour nous, et que nous pouvions agir en toute liberté, nos circulaires ont été approuvées. Genève devenait, puisque Paris était bloqué, le centre révolutionnaire de la province, en attendant que Lyon fasse son mouvement. Comme vous l'avez appris par les journaux, le mouvement de Lyon a été mal exécuté. Les hommes ont manqué totalement d'énergie et d'esprit révolutionnaire. Ces pauvres Français ne sont pas conspirateurs: on les prend comme des enfants. Encore dans cette affaire de Lyon qui devait être le début du soulèvement de la province, les amis de Bakounine et consorts ont fait échouer et compromis l'affaire; ils ont voulu en dehors de nous envoyer des affiches à Lyon qui ont été saisies en partie; d'autres avec des noms ont été placardées sur les murs de Lyon sans le consentement du Comité d'action de Lyon; ceci a tué les affaires bien organisées. Notre délégué Duval qui s'est même battu à Lyon nous a raconté que ces gens-là avaient fait du tort à la cause par leur stupidité et leur imprudence. Albert Richard à Lyon n'a rien fait, il est mal vu de la population ouvrière. Bref, malgré toute notre bonne volonté, nous avons échoué devant le manque d'énergie de la province : elle a laissé écraser Paris. Pourtant nous avions de quoi les seconder; nous avions des volontaires prêts à partir, même des officiers de grand mérite qui devaient se mettre à la tête des bataillons si le mouvement avait réussi. Enfin la Commune est tombée écrasée par la trahison et l'abandon. Nous avons continué à fonctionner, mais pour sauver nos amis de Paris échappés aux massacres. Nous avons envoyé à Paris l'un de nous avec des passeports suisses. ${ }^{2}$ Déjà bon nombre sont à Genève, d'autres viendront bientôt. Malon, Longuet et quelques autres sont cachés à Paris, nous les attendons ; ${ }^{3}$ Dereure

1 Anton Troussoff, typographe, membre de la section russe de l'Internationale à Genève.

2 Toute une série de passeports, d'actes d'origine et d'autres pièces furent établis au nom de membres peu connus de l'Internationale, à Genève, et employés pour permettre la fuite des communeux.

3 Longuet gagnera Londres; quant à Malon, il se trouvait déjà à Bâle, y attendant l'arrivée de sa compagne André Léo, au moment où Perret écrivait. Il arriva à Genève le 27 juillet. 
et Mégy sont à Londres, vous les verrez; ils ont été chez nous quelque temps. Frankel est encore à Genève. Notre chère Elise est menacée d'arrestation, mais nous veillons sur elle et nous voulons agir à Genève et dans la Suisse. ${ }^{1}$ Vous voyez, cher ami, que nous avons travaillé pour la cause. J'espère bientôt de vos nouvelles.

Je vous serre la main fraternellement.

Votre ami

H. Perret

Fonds Jung 900, I.I.S.G.

III

Genève, 26 novembre 1871

Cher ami,

Je t'écris seulement quelques lignes, dans quelques jours je t'écrirai plus longuement, car il se passe à Genève des choses très sérieuses; des menées indignes sont en train de chercher à nous diviser, on veut nous envahir à tout prix. Mais nous [sommes] prêts. Je suis peiné de voir la grande partie des proscrits français donner dans ces intrigues. Le mot d'ordre est de faire rejeter les résolutions de la Conférence de Londres. Guillaume a parlé, les hommes le suivent.

Tu recevras le journal officiel de cette nouvelle ligue, je te l'envoie. ${ }^{2} \mathrm{~J}$ 'aurai des nouvelles très importantes à te donner la semaine prochaine. Je te prie de faire prendre en considération les demandes que nous exprimons dans notre lettre, nous sommes en butte à des critiques qu'il faut éviter. ${ }^{3}$

Salue bien nos chers amis, Marx, Engels, Longuet, Rochat, Vaillant, Serailler, Martin, Eccarius.

Présente mes amitiés sincères à $M^{\text {me }}$ Jung, à ta fille Nelly et à toi je t'embrasse fraternellement.

H. Perret

Fonds Jung 903, I.I.S.G.

1 Le gouvernement de Versailles cherchait à obtenir l'extradition des proscrits en les accusant de crimes de droit commun. Le $1^{\mathrm{er}}$ juillet, la Légation de France à Berne demandait l'extradition de Frankel, le 12, celle de Mégy, de Dereure et d'uune femme portant le nom d'Elise, habitant avec le n[omm]é Outine». Se sentant menacés, nombre de proscrits passèrent de Suisse en Angleterre. Ce fut le cas de Dereure, de Mégy et, au mois d'août, de Frankel.

2 Il s'agit de La Révolution sociale, no 5, 23 novembre 1871. Dans ce numéro, le premier à porter le sous-titre "organe de la Fédération jurassienne", on trouve les résolutions du congrès de Sonvillier ainsi que l'annonce de la fondation de la Section de propagande et d'action socialiste révolutionnaire, composée des anciens membres de l'Alliance et d'une partie des réfugiés français.

3 Dans sa lettre "officielle», datée du même jour, Perret se plaignait, pour la seconde fois, de ne pas avoir reçu suffisamment d'exemplaires des résolutions de la Conférence de Londres et recommandait de ne correspondre qu'avec le secrétaire correspondant, pour éviter tout tiraillement. 
Association Internationale des Travailleurs

Genève, 9 juin 1872

Comité fédéral romand [tampon]

Au Conseil général de

l'Association Internationale des Travailleurs

Chers citoyens,

[....] Quant aux demandes formulées dans vos lettres, concernant les correspondants que nous avons en France, nous devons vous dire en premier lieu que nous n'avons donné des mandats que dans les villes suivantes: Toulon, Roanne, Grenoble et Lyon; à la Ciotat près Marseille, la section est organisée depuis longtemps et a dû correspondre directement avec le Conseil. Nous savons que divers membres de l'ancienne Alliance et de la Fédération jurassienne ont donné à tort et à travers des mandats, dans plusieurs villes de la France, d'abord au Creuzot. La plupart des proscrits qui étaient avec nous autrefois se sont tournés contre nous après [avoir] reçu des secours importants des sections de Genève. Dernièrement, de Toulon, on nous a prévenus de cesser momentanément toute correspondance; nous attendons des nouvelles. Vous recevrez plus tard un rapport sur nos relations avec la France. ${ }^{1}$ Voici quelques adresses avec qui nous sommes en relations: Busque, 57 rue de la Reine, Lyon; Labrosse à Chapelle de Girimchay, Saône et Loire, près Macon; Waguenère, chez $\mathrm{M}^{\mathrm{r}}$ Chemillière, rue Ste Anne Faubourg Mulssond, Maison Donjon, Roanne, Loire. Pour ces trois adresses il faut s'adresser au nom de Louis Guittat qui est un membre de notre Comité fédéral, et qui correspond avec ces trois citoyens. ${ }^{2}$ Pour Toulon: Amédée Suzan, rue du

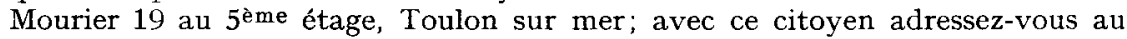
nom de $\mathrm{H}$. Perret, non le Comité fédéral. ${ }^{3}$ Voilà pour le moment les seules adresses que nous pouvons vous donner sûrement. Encore à Lyon et s'adresser au nom de H. Perret: $B^{\text {te }}$ Emmeler, graveur sur verre, chez $\mathrm{Mr}^{\mathrm{r}}$ Tanner à la Mouche Guillotière. Si vous n'avez pas d'adresse pour la Ciotat, en voici une: Duchanel, rue Couteau 5.

[....] Le citoyen Serailler dont nous ignorons l'adresse a bien voulu nous promettre quelques correspondances de la France pour être insérées dans l'Egalité; [il] nous a adressé une demande de lui donner deux adresses sûres pour ne pas éveiller les soupçons de la police des postes françaises; les voici et nous vous prions de les lui donner: Melle Aline Marcel, chez Mme Vve Humbert, Etuves 13 à Genève; l'autre à Mme Magnin, mercière, rue de Cornavin, Genève.

Extraits

Fonds Jung 879, I.I.S.G.

1 Ce rapport semble n'avoir jamais été envoyé.

${ }^{2}$ Louis Guittat, ferblantier lyonnais, réfugié à Genève après la Commune. Busque fut arrêté, à la fin de 1873 , et condamné pour tentative de reconstitution de l'Internationale à Lyon.

3 Dans la lettre du Comité fédéral romand au Conseil général, le 5 novembre 1871, Perret annonçait qu'ils avaient reçu l'adhésion d'une section de menuisiers à Toulon; conformément aux résolutions de Londres, le Comité fédéral avait envoyé un mandat à un correspondant toulonnais qui avait alors formé la section. 


\section{G. LEFRANÇAIS ET B. MALON A L. VERRYCKEN ${ }^{1}$}

\section{Cher Citoyen,}

Nous recevons une lettre de notre ami commun Guillaume de Neuchâtel, dans laquelle il nous fait connaître que, pas plus que la Fédération Jurassienne, la Fédération Belge n'entend laisser passer sans protestation les empiétements du Conseil général de Londres et les tendances autoritaires des résolutions arrêtées par la conférence du 17-23 septembre dernier.

Guillaume nous prie de plus, afin de vous édifier complètement sur la situation de l'Internationale en Suisse, de vous faire parvenir les impressions que nous en avons ressenties depuis notre arrivée ici, c'est-à-dire depuis la fin de Juillet dernier.

Désireux comme tous que la lumière se fasse, nous nous empressons de vous adresser ces impressions. [Lorsque nous arrivâmes tous deux à Genève, quelque peu brisés de la chute de notre chère Commune,] nous aspirions, non au repos ni à l'inaction, ce qui n'eût été ni digne de l'idée que nous avons tenté de réaliser, ni digne de véritables socialistes, mais du moins à un calme qui nous permît de nous recueillir et de ressaisir nos idées, afin d'étudier sur quel terrain désormais nous devions continuer notre action. Nous avions aussi décidé de ne tenir aucun compte des froissements de la lutte précédente et de nous occuper exclusivement de l'avenir.

[Nous arrivions] enfin [résolus à ne point tenir compte des différends qui partagent depuis trop longtemps les sections de la Suisse Romande, différends dont nous avions entendu vaguement parler et qui ne reposent d'ailleurs que sur des questions de personnes dont à l'un de nous (Lefrançais) les noms seuls étaient] à peine [connus.

Dans ces dispositions nous résolûmes de ne prendre parti ni pour un groupe ni pour l'autre, les personnalités d'Outine et de Bakounine, seules en jeu dans cette affaire, ne nous paraissaient pas devoir occuper le temps si précieux de ceux qui] dévouent leur existence précisément à la réalisation d'un état social où les principes seuls seront en jeu.

[A peine arrivés, nous fûmes naturellement vite tenus au courant des divisions en question, mais, nous devons le dire, seulement par les adversaires de l'ancienne Alliance, dont les amis au contraire se tinrent à notre égard dans la plus grande réserve.

Mais un fait douloureux ne tarda pas à devenir évident pour nous: Malgré la liberté dont jouissent les Genevois; malgré tous les moyens dont ils disposent - liberté de la presse, liberté de réunion, d'association - l'Internationale n' a en réalité aucune existence intellectuelle ici: ni réunions, ni conférences, ni discussion de

1 Cette lettre a été reproduite partiellement par Guillaume (op. cit., t. II, pp. 216-217); nous avons indiqué entre crochets les passages déjà publiés. Les suppressions ne portent pas sur l'essentiel, mais la version donnée par Guillaume laisse au lecteur l'impression d'une lettre purement spontanée, alors qu'elle a été, en fait, écrite sur demande de Guillaume lui-même, afin d'influer sur le congrès de la Fédération belge, qui se tint à Bruxelles, les 24 et 25 décembre 1871 . 
principes. La plupart des adhérents sont dans l'ignorance absolue des principes de l'Internationale et du but qu'elle poursuit. Chacun se contente de dire: Je suis de l'Internationale! Mais encore une fois, rien de sérieux - les intelligents, dégoûtés, s'en retirent ou en sont exclus par les comités qui, seuls, gouvernent et dirigent les sections, qui se réunissent seulement une fois par mois à peine! Pas de cercles d'études sociales où tous puissent venir s'instruire des questions à résoudre. - Les choses se passaient autrement en France où la période de combat avait été précédée et amenée par une longue sérieuse période de propagande et d'étude et ces souvenirs nous faisaient d'autant plus ressentir le vide existant dans les sections genevoises.

Quelques amis, et nous avec eux, sommes entrés alors dans la section centrale pour tenter de lui imprimer un mouvement plus sérieux et plus actif, mais les pasteurs y ont mis ordre, comme vous le verrez par le compte-rendu de la dernière assemblée générale des sections,] que vous trouverez dans le numéro de la Révolution sociale, que nous joignons à cette lettre. ${ }^{1}$ [On nous a exclus tout simplement comme agents de l'ancienne Alliance et de Bakounine, à qui Lefrancais n'a jamais parlé,] qu'il a vu par hasard une ou deux fois à la Tribune des Peuples en 1849! (il y a 22 ans!). [Quant à Malon, il connaissait Bakounine, mais jamais entente ne fut établie entre eux.]

Dans de telles circonstances, force nous a été de nous rendre à l'évidence et de prendre un parti. C'est alors que nous sommes devenus membres de la Fédération Jurassienne, à laquelle a adhéré la section de propagande et d'action socialiste-révolutionnaire, fondée par des réfugiés français et autres et dans laquelle on nous a reçus.

Dans cette Fédération au moins et à l'aide de la section de Genève, nous allons avec nos amis, laissant de côté l'élément genevois, sur lequel nous n'aurions aucune action possible et qui nous paraît réfractaire à toute pensée d'études sérieuses, nous allons tenter de continuer à travailler pour nos amis de France qui en ont tant besoin, désorientés qu'ils sont, en essayant de les rallier et de les grouper. Plus que jamais à notre avis la propagande socialiste est devenue une nécessité révolutionnaire.

Enfin nous sommes décidés à joindre tous nos efforts à ceux qui veulent provoquer le plus promptement possible un congrès général qui mettra ordre à toutes les questions, secondaires, mais d'autant plus dangereuses, qu'elles seraient de nature, si elles se prolongeaient, à faciliter les projets de gens qui nous paraissent trop enclins à faire servir l'association à des intérêts politiques, que cette association a précisément pour but spécial d'éliminer pour l'avenir et qui sont tout à fait contraires à l'idée du fédéralisme.

Nous sommes sûrs que toutes ces graves questions, et surtout celle qui se pose par la XVme résolution de la conférence du 17-23 septembre, seront sérieusement débattues au congrès régional des internationaux de la Belgique, dont nous attendrons les résolutions avec impatience, pensant que vous n'oublierez pas que ce [que] vous allez décider aura une grande influence sur les destinées de l'Internationale si prouvée en ce moment et que vos délibérations s'en ressentiront.

Salut et Solidarité.

G. Lefrançais

membre de la Fédération Jurassienne

1 Le no 7, du 7 décembre 1871, contenant le compte rendu, signé de Lefrançais, de l'assemblée générale des sections genevoises du 2 décembre 1871. 
Section de propagande et d'action socialiste-révolutionnaire

à Genève

1 place de Rive chez M. Oettinger

\author{
B. Malon \\ Membre de la section de propagande et de \\ la Fédération Jurassienne \\ 7 rue de la Croix d'or \\ Genève
}

Collection Descaves, I.I.S.G.

VI

NICOLAS OUTINE A KARL MARX

Genève, le 1 er novembre [1872]

[....] Le deuxième, Woldemar Ozeroff, est un des plus vilains coquins de l'Alliance; il a été aide-de-camp de Bakounine dans sa campagne lyonnaise et un jour il est venu s'excuser auprès de moi pour tout le passé, me demandant que je leur trouve 100 mille francs pour sauver la France (c'était pendant la guerre), il se contenta de 100 francs que j'ai eu la bêtise de donner alors - j'en confesse! non pour sauver la France, mais pour donner à manger à ceux qu'ils ont réussi de jeter en prison à Lyon. Plus tard, sous la Commune, il vint à nous, jurant qu'il veut agir d'accord avec nous pour préparer l'insurrection à Lyon; il nous a trahis, en rapportant tout à l'Alliance et en agissant d'accord avec G. Blanc et Richard dans l'envoi des proclamations - signées de plusieurs noms sans autorisation -, avec Albert Leblanc de Genève à Lyon. Alb. Leblanc était arrêté, dénoncé on ne sait par qui (je ne veux pas dire que ce fût par Ozeroff, non) et la police se trouvait en possession des documents, sur lesquels les signatures compromettaient beaucoup de personnes à leur insu. C'est cette affaire qui a fait échouer l'insurrection à Lyon et c'est dans cette affaire qu'Ozeroff joua un rôle de coquin.

[..... Ce matin on m'a amené un délégué français de Paris et de Lyon: le pauvre homme est envoyé ici pour se renseigner, pour comprendre avec qui ils doivent marcher: si c'est avec New-York, ou Genève, ou Société jurassienne, ou Ranvier, Vaillant et Cie? - Je lui ai dit que New-York et Genève ne faisaient qu'un, et que les deux autres étaient adversaires de l'Internat[iona]le. Il viendra aujourd'hui encore pour m'embêter, puis il ira au $\mathrm{T}$ [emple] unique et demain soir il sera encore chez moi avec les membres du Comité fédéral. Si j'apprends de lui quelque chose d'intéressant, je ne faillirai pas de le communiquer dans une lettre à la sœur Tussy. En attendant, j'ai appris déjà ceci: 1- Ranvier, Vaillant et $\mathrm{C}^{\mathrm{i}}$ se préparent à lancer un manifeste expliquant pourquoi ils se sont retirés avant le Congrès (!!), et annonçant la constitution d'une société politique. 2Avant le Congrès, Ranvier a écrit à Paris en engageant les Parisiens à se méfier de Séraillier et traitant Séraillier de réactionnaire! 3- Avant le Congrès aussi, un délégué, un Polonais à une jambe, est venu de la part de la Société jurassienne pour engager les Internationaux de Paris à se déclarer contre Londres et à adhérer à la Société jurassienne pour former une contre-Internationale, sur ce, il fut mis à la porte... Ma foi! cela soulève le cœur toutes ces turpitudes!

Extraits

Fonds Marx-Engels D 4349, I.I.S.G. 
VII

VICTOR JACLARD A KARL MARX

Zeltweg, zum Thalegg, Hottingen Zurich, 3 janvier 1873

\section{Citoyen,}

Je vous suis très reconnaissant des renseignements que vous avez eu l'obligeance de me faire parvenir par l'intermédiaire de ma femme.

Avouons que les bonapartes ne sont pas de si mauvaises gens qu'on veut bien le dire, puisqu'ils récompensent si largement la haine de leurs ennemis. La mauvaise fortune les a bien changés et les a rendus tout à fait débonnaires!

Et puis c'est un système de gouvernement comme un autre que de gouverner avec ses adversaires. Nous connaissions depuis longtemps "la république sans républicains", pourquoi n'essayerait-on pas de "l'empire sans impérialistes". Nous avons vu et nous voyons encore aujourd'hui une république sous laquelle les monarchistes massacrent les républicains; nous aurions un empire qui chargerait les républicains et les socialistes de fusiller ou de jeter à Mazas tout ce qui peut être soupçonné de bonapartisme. M. Gambetta prétend s'être très bien trouvé de cette manière de gouverner et $M$. Thiers affirme que c'est la seule possible. Pourquoi les bonapartes à qui les autres systèmes ont si peu réussi ne songeraient-ils à appliquer cette méthode nouvelle, aujourd'hui tout à fait à la mode? Cette hypothèse, une fois admise, nous expliquera bien des choses. Par exemple voici Ducrot surpris en train de fabriquer des listes de suspects. On se récrie, on demande des explications. Les républicains ont bien tort de s'alarmer. C'est pour eux qu'on travaille. Cette prétendue liste de suspects n'est autre chose que la liste du futur gouvernement bonapartiste.

En ce qui me concerne personnellement, il est donc vraisemblable qu'un beau matin, je trouverai sur ma table un pli aux armes de Chilshurst, ${ }^{1}$ et contenant ces mots: "Citoyen, convaincu que personne ne saurait être plus hostile que vous l'êtes à la cause de l'ordre en général et à la cause bonapartiste en particulier, nous, empereur des Français, vous considérons comme tout à fait apte à prendre part à la direction de nos affaires. En conséquence nous nous permettons de mettre à vos pieds un des portefeuilles disponibles, ne vous soumettant qu'une seule condition, c'est qu'au lieu de diminuer, votre haine ne fera que croittre tous les jours, si c'est possible, etc.".

Mais de même que le vrai n'est pas toujours vraisemblable, le vraisemblable n'est pas toujours vrai.

Je ne me rappelle pas avoir reçu un pli de la sorte. Il eût cependant été convenable que je fusse tout au moins prévenu de ma nomination. Mais ces bonapartistes manquent de tout savoir vivre.

Cependant, si je fais appel à mes souvenirs éloignés, il me revient à l'esprit une visite étrange que je reçus il y a un an environ, je ne saurais préciser exactement la date.

Je connaissais Richard assez vaguement depuis plusieurs années, n'ayant avec lui que des relations indirectes. Au lendemain du 4 septembre, me rendant de Genève à Paris, je m'arrêtai à Lyon. Je voulus profiter de mon passage pour essayer de poser les premières bases d'une ligue du midi ayant à sa tête une

1 Lire Chislehurst, lieu où s'était réfugié l'ex-empereur Napoléon III. 
convention révolutionnaire. Richard qui m'avait accueilli à bras ouverts et m'avait offert dans sa famille une hospitalité tout à fait cordiale, m'appuya dans mon projet d'organisation et la section internationale, convoquée sur sa demande, me donna également tout son concours. Le mouvement était en bonne voie quand je partis pour Paris en compagnie de Richard et d'Andrieux, l'avocat, tous trois délégués par le peuple lyonnais. Quelques jours après, Richard et Andrieux me quittaient pour regagner Lyon, ce dernier emportant dans sa poche sa nomination de procureur.

Je rappelle ces faits pour préciser la nature de mes relations, devenues un moment assez intimes, avec Richard. Cette circonstance me permit de compléter mon jugement sur celui-ci. Je reconnus en lui un mélange de certaines qualités, quelques-unes assez brillantes, avec une série de défauts d'un caractère capital: une ambition extrêmement personnelle, une vanité dépassant toute limite, un esprit dépourvu de tout équilibre.

Je ne le revis plus depuis lors. Un soir - j'étais alors à Berne - on m'annonça un visiteur; je reconnus le père de Richard. M'étant évadé depuis peu et me trouvant tout à fait isolé à Berne, où quelques proscrits, Protot et Dessesquelle entre autres n'étaient arrivés que depuis quelques jours, j'étais dans une ignorance absolue concernant les bruits qui couraient sur Richard, ou du moins je n'y avais accordé aucune attention, les considérant comme des calomnies pures et simples.

Je reçus donc mon visiteur très amicalement et l'interrogeai sur la situation politique de la ville de Lyon, sur l'état des esprits, les dispositions de l'armée, etc. La réponse fut que l'armée était dévouée corps et âme aux bonapartistes, que le peuple de son côté considérait comme sa seule ressource de s'allier à l'empire pour écraser la bourgeoisie et fonder le socialisme. Comme je lui exprimais ma profonde stupéfaction et renouvelais mes questions d'une manière de plus en plus pressante pour savoir sur quelles bases il appuyait son jugement, ses réponses devinrent très embarrassées, et il prit l'air d'un homme engagé dans une mare dont il ne saurait plus comment se dépêtrer. Comme il se disposait à s'en aller, je lui donnai, pour le lendemain, un rendez-vous où nous devions trouver réunis quelques proscrits. Il prétexta des raisons d'affaires qui l'obligeaient à se rendre à Bâle et se retira en me promettant de revenir me voir à son retour. Le lendemain, je racontai l'incident à Protot, Dessesquelle, etc. Ils me parlèrent des accusations dont Richard était l'objet. Depuis j'eus entre les mains la brochure bien connue se terminant par ces mots solennels: "Vive l'empereur".

Il va sans dire que le père Richard ne revint plus. J'appris que Richard était lui-même venu à Berne, où il s'était rencontré, dit-on, avec Bastélica. Mais je ne l'ai pas vu.

De près ou de loin, directement ou indirectement, je n'ai donc eu, depuis le 10 septembre, aucun rapport avec Richard ou ses complices, hormis cette visite dont je vous ai donné les détails.

Bien que les bonapartistes aient souvent fait leurs preuves, cependant je ne puis m'empêcher de constater l'audace de ce procédé qui consiste à faire de mon nom un pareil usage. Une telle fourberie place M. Richard au dernier rang des policiers.

Je ne vous parle ni de Gasp[ard] Blanc, ni de Sholh, ${ }^{1}$ ni d'autres, qui me sont tout à fait inconnus. J'ai dû rencontrer à Lyon Gasp. Blanc, mais sans le remarquer.

1 Lire Chol, membre de l'Internationale lyonnaise et participant au mouvement ouvrier de mars 1871. 
Vient maintenant la question de savoir si le fait mérite les honneurs de la publicité. Je professe une assez grande indifférence pour des insultes qui ont une telle origine, et il me répugne d'occuper le public d'un cancan aussi grotesque.

Cependant il peut être utile de le faire. Mes amis de Londres peuvent s'en rendre compte beaucoup mieux que moi. J'accepterais donc volontiers leur avis en cette occasion. D'un autre côté, j'ignore dans quelle mesure je puis me permettre de faire usage des renseignements que vous avez bien voulu me communiquer.

En ce qui me concerne, il me suffit de connaître l'opinion de nos amis; je me serais tout autrement ému, si votre lettre ne m'avait convaincu que cette calomnie n'a soulevé en eux que l'indignation et le dégoût. Je leur en sais beaucoup de gré et n'attendais du reste pas moins de leur bonne amitié. Je professe pour mes amis une très grande et très profonde estime et il me faudrait des preuves bien frappantes pour me permettre d'élever contre eux le moindre soupçon. Je croirais les offenser en ne comptant pas sur une absolue réciprocité de leur part.

Je suis en particulier très reconnaissant à Longuet, bien que vous ayez pu penser que sa vivacité ait compromis le succès du plan que vous dictait un intérêt plus général.

Enfin, veuillez accepter pour vous-même mes très sincères remerciements et croire à mes meilleurs sentiments.

V. Jaclard

P.S. Puis-je me permettre de vous prier de me rappeler au souvenir de Mesdemoiselles Marx, avec lesquelles j'ai passé quelques heures si agréables il y a plusieurs années. Je serais très heureux d'avoir des nouvelles de mon excellent ami Lafargue ou tout au moins d'avoir son adresse.

J'ai oublié d'ajouter que vous pouviez faire de ma lettre l'usage que vous jugeriez convenable.

V.J.

Fonds Marx-Engels D 2457, I.I.S.G. 


\section{LIBERTY, EQUALITY,SOLIDARITY, FRATERNITY.}

Anniversary of the 18th of IIarch, 1871 (COMMUNALIST REVOLUTION OH PARIS.)

The International Federal (French) Section of 1871 invites Democrats of all countries to assist at a COMMEMORATIVE MEETING,

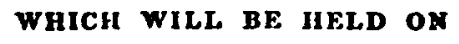

Monday, March 18, 1872,

New Hall of Science,

CLUB AND INSTITUTE,

142, OLD STREET, CITY RD.

THE CRAIK WILL BE TAKEN BY

OIIIZEN DUPONT,

RX-MEMBER OF THE COMAUNE OD PARIS.

Speeches delivered in French will be translated into Einglish.

\section{ADMISSION TWOPENCE.}

Orators:

LANDECK, Delegate' from the Comnune of Marseilles.

IEVERDAYS.

COMBATZ, Chef de Legion.

VESINIER, Member of the

('ommune.
Members of the Cisummission :

A. DUPON'.

B. LANDECK.

LAROQUE, Memler of the Central Committec.

E. E. METARD.

DEBORD. 